\title{
Period changes in W UMa-type eclipsing binaries: DK Cygni, V401 Cygni, AD Phoenicis and Y Sextantis ${ }^{\star}$
}

\author{
M. Wolf ${ }^{1}$, P. Molík ${ }^{1}$, K. Hornoch ${ }^{1}$, and L. Šarounová ${ }^{2}$ \\ 1 Astronomical Institute, Charles University Prague, CZ-180 00 Praha 8, V Holešovičkách 2, Czech Republic \\ e-mail: wolf@mbox.cesnet.cz \\ 2 Astronomical Institute, Academy of Sciences of Czech Republic, CZ-251 65 Ondřejov, Czech Republic
}

Received July 21; accepted September 11, 2000

\begin{abstract}
We present a period analysis of the four wellknown W UMa-type eclipsing binaries DK Cyg $(P=$ 0.47), V401 Cyg (0.58), AD Phe (0.38) and Y Sex (0.42). Several new times of minimum light, recorded photoelectrically, have been gathered. Analysis of all available eclipse timings of the DK Cygni and V401 Cygni has confirmed a significant increase in period of $1.1510^{-10}$ and $1.4810^{-10}$ day cycle ${ }^{-1}$, respectively. A simultaneous solution of the $B, V$ and $R$ light curves was computed for V401 Cyg using the Binary Maker 2.0 synthetic lightcurve software. This solution indicates that V401 Cyg is in contact with a filing factor of $46 \%$.

The period of AD Phe seems to be constant. Period changes of Y Sex could be explain by a light-time effect caused by a third body in an eccentric orbit with a period of 58 years.
\end{abstract}

Key words: stars: binaries: eclipsing — stars: individual: DK Cyg - stars: individual: V401 Cyg — stars: individual: AD Phe — stars: individual: Y Sex — stars: fundamental parameters

\section{Introduction}

W UMa-type eclipsing binaries are an important source of information about the evolution of close binary systems. In this paper, we report results of our observations monitoring W UMa-type eclipsing binaries with period changes (Wolf et al. 1996; Molík \& Wolf 1998). In particular, four objects with the similar brightness ( $V \simeq 10 \mathrm{mag}$ ), namely DK Cyg, V401 Cyg, AD Phe and Y Sex, are analysed here.

Send offprint requests to: M. Wolf

* Some of the observations reported in this paper were obtained at the South Africa Astronomical Observatory, Sutherland, South Africa.
With the exception of DK Cyg, which was found to be a variable star by Guthnick \& Prager (1927), the other variables were discovered by Cuno Hoffmeister. They all are well-known close binaries and their long epoch of observations gives us the possibility to study many interesting phenomena in these systems. The purpose of the present work is to present new photoelectric times of minimum light, to make a period study of these systems and to derive new light elements.

\section{Observations and period analysis}

In order to enlarge the number of times of minimum light, new observations for all three systems were carried out. Our new photoelectric photometry was performed at three observatories with the aim of securing at least two wellcovered minima for each variable:

- South Africa Astronomical Observatory (SAAO), Sutherland, South Africa: $50 \mathrm{~cm}$ Cassegrain telescope with Johnsons $U B V$ filters and classical photometer;

- Ondřejov Observatory, Czech Republic: 65 cm reflecting telescope with CCD camera SBIG ST-8 and Cousins $R$ filter;

- private observatory of K.H. at Lelekovice, Czech Republic: $35 \mathrm{~cm}$ Newtonian telescope with CCD camera SBIG ST-6V and $R$ filter.

Photoelectric observations at SAAO were obtained with the modular photometer utilizing a Hamamatsu GaAs R943-02 photomultiplier during two weeks in August 1999. Each observation of an eclipsing binary was accompanied by observation of a local comparison star. The photoelectric measurements were done in the $U B V$ filters of the Johnson's photometric system with a $10 \mathrm{~s}$ integration time. All observations were reduced to the Cousins E-region standard system (Menzies et al. 1989).

The CCD measurements in Ondřejov were done using the standard Cousins $R$ filter. Flat fields for the reduction 
Table 1. New precise times of minimum light

\begin{tabular}{lllccc}
\hline System & $\begin{array}{l}\text { JD Hel.- } \\
2400000\end{array}$ & $\begin{array}{l}\text { Error } \\
\text { [day] }\end{array}$ & $\begin{array}{c}N \\
\text { Method } \\
\text { Filter }\end{array}$ & Observatory \\
\hline DK Cyg & $47963.662^{*}$ & 0.001 & 15 & $\mathrm{H}$ & Hipparcos \\
& $47963.896^{*}$ & 0.001 & 16 & $\mathrm{H}$ & Hipparcos \\
& $48297.616^{*}$ & 0.001 & 14 & $\mathrm{H}$ & Hipparcos \\
& $48302.793^{*}$ & 0.001 & 10 & $\mathrm{H}$ & Hipparcos \\
& 51379.4817 & 0.0001 & 54 & $\mathrm{CCD},-$ Jindř. Hradec \\
& 51749.4447 & 0.0001 & 76 & $\mathrm{CCD}, R$ & Lelekovice
\end{tabular}

$\begin{array}{rlllll}\text { V401 Cyg } 48254.0593 & 0.0005 & 10 & \mathrm{H} & \text { Hipparcos } \\ 51333.46434 & 0.0001 & 88 \mathrm{CCD}, R & \text { Ondřejov } \\ 51391.44435 & 0.0001 & 54 \mathrm{CCD}, R & \text { Ondřejov } \\ 51394.360 & 0.0005 & 15 \mathrm{CCD}, R & \text { Ondřejov } \\ 51680.48045 & 0.00007 & 70 \mathrm{CCD}, R & \text { Ondřejov } \\ 51738.4647 & 0.0001 & 75 \mathrm{CCD}, R & \text { Ondřejov }\end{array}$

$\begin{array}{cllccc}\text { AD Phe } & 48126.759^{*} & 0.001 & 11 & \mathrm{H} & \text { Hipparcos } \\ & 48480.4675 & 0.001 & 6 & \mathrm{H} & \text { Hipparcos } \\ & 48480.658^{*} & 0.001 & 18 & \mathrm{H} & \text { Hipparcos } \\ & 48540.496^{*} & 0.001 & 12 & \mathrm{H} & \text { Hipparcos } \\ & 48540.686^{*} & 0.001 & 16 & \mathrm{H} & \text { Hipparcos } \\ 51412.5292 & 0.0005 & 13 & \text { pe, } B V & \text { SAAO } \\ & 51417.46805 & 0.0001 & 13 & \text { pe, } B V & \text { SAAO } \\ & 51418.41447 & 0.0001 & 37 & \text { pe, } B V & \text { SAAO }\end{array}$

Y Sex $48068.526^{*} \quad 0.001 \quad 10 \quad$ H $\quad$ Hipparcos $51655.4420 \quad 0.0005 \quad 44 \mathrm{CCD}, R \quad$ Lelekovice $51663.4184 \quad 0.0001 \quad 66 \mathrm{CCD}, R \quad$ Lelekovice

* Normal minimum.

of the CCD frames were routinely obtained from exposures of regions of the sky taken at dusk or dawn. Several comparison stars were chosen on the same frame as the variables. During the observations, no variations in the brightness of these stars exceeding the possible error of measurements (typical $\sigma \simeq 0.005 \mathrm{mag}$ ) were detected. No correction was performed for differential extinction, due to the proximity of the comparison stars to the variable and the resulting negligible differences in the air mass.

The new times of primary and secondary minimum and their errors were determined using the least squares fit to the data by the bisecting chord method. These times of minimum are presented in Table 1. From the Hipparcos photometry (Perryman 1997), we were able to determine several additional moments of minimum light. They are given also in Table 1 . In this table $N$ stands for the number of measurements used for the determination of minimum time.

Period changes in all systems were studied by means of an $\mathrm{O}-\mathrm{C}$ diagram analysis. We have collected all reliable times of minimum light gathered from the literature, from current databases of AAVSO, BAV and BBSAG observers as well as from the Besançon Double and Multiple Star Database. We employed the following data reduction procedure. All photoelectric times of minimum were used with a weight of 10 in our computation. The current less precise measurements were weighted with a factor of 5 , while the earlier visual and photographic times of minimum were given a weight of 1 due to the large scatter in these data.

\section{DK Cyg}

The eclipsing binary DK Cyg (also BD $+33^{\circ} 4304$, HIP 106574, AN 1.1927, FL 3231; $\alpha_{2000}=21^{\mathrm{h}} 35^{\mathrm{m}} 02.7^{\mathrm{s}}$, $\left.\delta_{2000}=+34^{\circ} 35^{\prime} 45.4^{\prime \prime}, V_{\max }=10.30 \mathrm{mag} ; \mathrm{Sp} . \mathrm{A} 8 \mathrm{~V}\right)$ is a well-known W UMa-type binary with a period of about 0.4707 days. It was discovered to be a variable by Guthnick \& Prager (1927). The first photoelectric observations were made by Hinderer (1960). Binnendijk (1964) in his photometric study derived linear light elements:

Pri. Min. = HJD $2437999.5838+0.47069055 \cdot E$

and found the change of the secondary minimum depth. The period study of DK Cyg was published by Paparo et al. (1985). They showed that the orbital period was increasing and calculated the first parabolic light elements:

Pri. Min. = HJD 2437999.5828

$$
+0.47069066 \cdot E+5^{\mathrm{d}} 3910^{-11} E^{2} \text {. }
$$

The next photoelectric study was published by Awadalla (1994) who refined the light elements:

Pri. Min. = HJD 2437999.58249

$$
+0.47069073 \cdot E+5.7610^{-11} E^{2}
$$

and confirmed the light curve variability. See also that paper for further details and a historical review of other observations. The first spectroscopic study and radial velocity curve analysis was presented by Rucinski \& Lu (1999) who found $q=m_{2} / m_{1}=0.325$. Recently, updated linear ephemeris of DK Cyg was presented by Kiss et al. (1999):

Pri. Min. = HJD $2451000.0999+0$ d $47069290 \cdot E$.

All photoelectric times of minimum light published in Hinderer (1960), Binnendijk (1964), Paparo et al. (1985), Agerer (1988, 1990, 1992), Awadalla (1994), Agerer \& Hübscher (1995, 1996) and Hegedüs et al. (1996), as well as new timings given in Table 1 were incorporated in our analysis. One additional time of primary minimum was obtained by J. Štrobl at the Jindřichův Hradec Observatory, Czech Republic, with a CCD camera SBIG ST6 and a $16 \mathrm{~cm}$ Newtonian telescope. Other numerous visual and photographic estimations obtained by the AAVSO, BAV and BBSAG observers were used with less weight. A total of 95 times of minimum were incorporated in our analysis. Using the method of least squares we derived the following light elements with a quadratic term:

Pri. Min. = HJD $2437999.5825+$

$$
\begin{gathered}
\pm 0.0004 \\
0.47069064 \cdot E+5.7510^{-11} E^{2} . \\
\pm 0.00000008 \quad \pm 0.03
\end{gathered}
$$




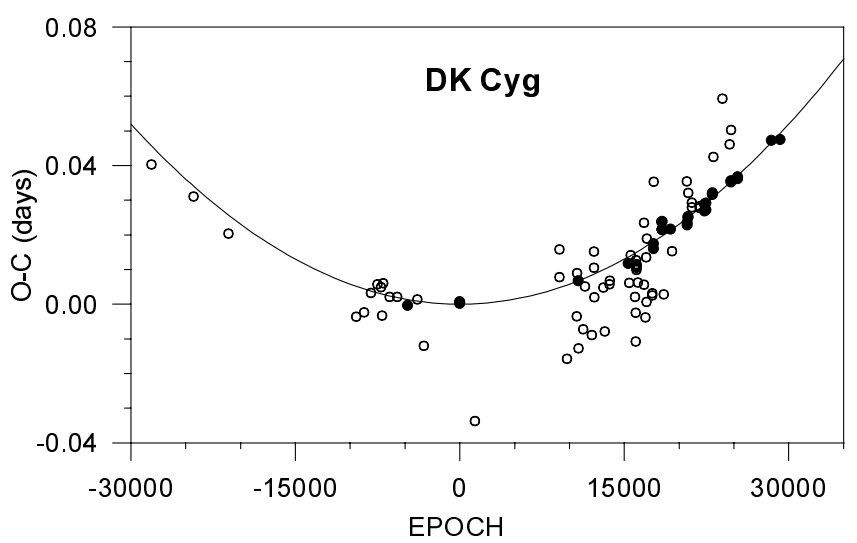

Fig. 1. O-C residuals for the times of minimum of DK Cyg with respect to the linear light elements. The continuous curve represents the parabolic approximation. The individual photoelectric times are denoted by dots, photographic or visual estimations by circles

The $\mathrm{O}-\mathrm{C}$ residuals for all times of minimum with respect to the linear ephemeris are shown in Fig. 1. The non-linear fit, corresponding to the light elements given above, is plotted as a continuous curve. The period increase resulting from these elements is $\mathrm{d} P / \mathrm{d} E=1.1510^{-10}$ day/cycle or $8.9210^{-8}$ day/year or 0.8 seconds/century. Because the long-term increase in orbital period is usually explained by mass transfer from the secondary to the primary component, we calculated the value of this supposed mass transfer for DK Cyg. If the mass transfer is conservative with no magnetic field we determine the mass transfer rate $\mathrm{d} M / \mathrm{d} t=2.8610^{-8} M_{\odot} /$ year for a total mass of the system $M_{1}+M_{2}=2.0 M_{\odot}$ and the mass ratio $q=0.325$ (Rucinski \& Lu 1999).

\section{V401 Cyg}

The eclipsing binary V401 Cyg (also BD $+30^{\circ}$ 3592, HIP 95816, AN 88.1929, FL 2747, P1824; $\alpha_{2000}=19^{\mathrm{h}} 29^{\mathrm{m}} 20.3^{\mathrm{s}}, \delta_{2000}=+30^{\circ} 24^{\prime} 28.5^{\prime \prime}, V_{\max }=$ 10.8 mag; Sp. F0) is a relatively well-known W UMa-type binary with period of about 0.58 days. It was discovered to be a variable by Hoffmeister (1929) and for a long time it was misclassified as RR Lyrae type variable with period 0.2895 days. Lurye (1947) found its eclipsing binary nature and derived the light elements with the correct period:

Pri. Min. = HJD $2428838.382+0.58271446 \cdot E$.

Early photoelectric observations were obtained by Spinrad (1959) in June 1958 at Berkeley. He confirmed the eclipsing nature of its variability and found a colour index $B-V=0.27$ mag. Next $B, V$ photoelectric photometry was obtained by Purgathofer (1964) at Lowell observatory during several nights in 1961. He found the value of $B-V=0.36 \mathrm{mag}$ and revealed the remarkable increase

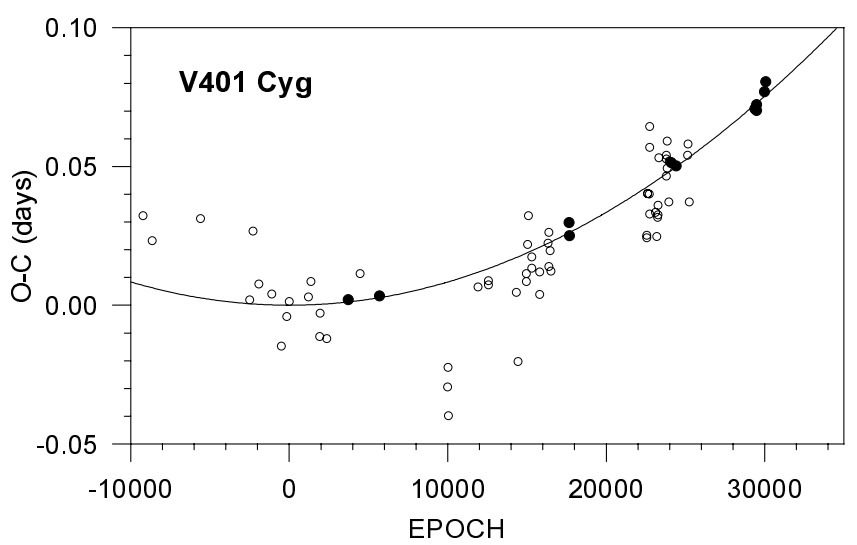

Fig. 2. O-C graph for V401 Cyg. See legend for Fig. 1

in its period. This led him to the calculation of the new quadratic ephemeris:

Pri. Min. = HJD 2434215.693

$$
+0.58271901 \cdot E+2.5510^{-10} E^{2} \text {. }
$$

A period study of V401 Cyg was presented also by Herczeg (1993), who reported several photoelectric times of minima and confirmed that the period was still increasing. He also derived the corrected quadratic light elements:

$$
\begin{aligned}
\text { Pri. Min. }= & \text { HJD } 2434215.695 \\
& +0.5827187 \cdot E+1.1010^{-10} E^{2} .
\end{aligned}
$$

All times of minimum light given in Herczeg (1993, his Table 1) as well as new timings were incorporated in our analysis. Using the method of least squares we calculated the following light elements with a quadratic term:

Pri. Min. = HJD 2434215.6924

$$
+0.58272061 \cdot E+7^{\mathrm{d}} .3910^{-11} E^{2} \text {. }
$$

The $\mathrm{O}-\mathrm{C}$ residuals for all times of minimum with respect to the linear ephemeris are shown in Fig. 2. The non-linear fit, corresponding to the light elements given above is plotted as a continuous curve. The period increase $\mathrm{d} P / \mathrm{d} E=$ $1.4810^{-10}$ day/cycle or $9.2610^{-8}$ day/year or 0.8 seconds/century resulting from these elements is relatively large for a W UMa-star. Assuming the value of mass ratio $q=0.3$, resulting from our light curve analysis, and a total mass $M_{1}+M_{2}=1.8 M_{\odot}$, one can obtain a mass-exchange rate for this binary $\mathrm{d} M / \mathrm{d} t=2.0310^{-8} M_{\odot} /$ year.

The $B$ and $V$ light curves of V401 Cyg published by Purgathofer (1964), as well as our $R$ light curve, have been used simultaneously for the determination of the geometric and photometric elements using the Binary Maker 2.0 reduction software (Bradstreet 1993). This program, based on the Wilson-Devinney algorithm, is used for a preliminary analysis of light curves by graphically producing models of close binaries. First, the observed points ordered in phase were combined into 177 normalized binned points in each colour. Based on the GCVS's spectral classification of the primary component, F0, and the colour index $B-V=0.36$ mag (Purgathofer 1964), the temperature 
Table 2. Synthetic light curve parameters for V401 Cyg

\begin{tabular}{ccc}
\hline Parameter & Unit & Value \\
\hline$\lambda_{B}$ & $\AA$ & 4400 \\
$\lambda_{V}$ & $\AA$ & 5500 \\
$\lambda_{R}$ & $\AA$ & 6900 \\
$x_{1 B}=x_{2 B}$ & & 0.74 \\
$x_{1 V}=x_{2 V}$ & & 0.60 \\
$x_{1 R}=x_{2 R}$ & & 0.48 \\
$g_{1}=g_{2}$ & & 0.32 \\
$T_{1}$ & $\mathrm{~K}$ & 6700 \\
$T_{2}$ & $\mathrm{~K}$ & 6650 \\
$i$ & $\mathrm{deg}$ & 77 \\
$\Omega_{1}=\Omega_{2}$ & & 2.38 \\
$q$ & & 0.30 \\
$L_{3}$ & & 0 \\
$r_{1}, r_{2}$ (pole) & & $0.474,0.281$ \\
$r_{1}, r_{2}$ (side) & & $0.515,0.296$ \\
$r_{1}, r_{2}$ (back) & & $0.547,0.348$ \\
fill out & & $46 \%$ \\
\hline
\end{tabular}

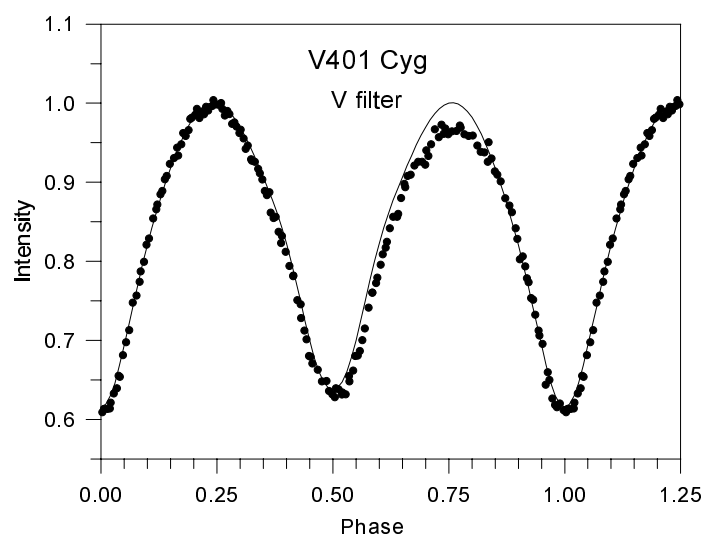

Fig. 3. $V$ light curve of V401 Cyg obtained by Purgathofer (1964). The continuous line represents the light-curve solution with parameters given in Table 2 . The shift of the secondary minimum from the phase 0.5 is clearly visible

was adopted to be $T_{1}=6700 \mathrm{~K}$. The other adopted parameters are the gravity-darkening coefficients $g_{1}=g_{2}$, the albedoes $A_{1}=A_{2}$ and the linear limb-darkening coeficients $x_{1}$ and $x_{2}$. We adjusted the following parameters: the mass ratio $q=M_{2} / M_{1}$, the potential function $\Omega_{1}, \Omega_{2}$, the temperature of the secondary component $T_{2}$, and the orbital inclination $i$. The final solution is given in Table 2; the uncertainty in the inclination is about \pm 2 degrees and the difference in temperature is known to about $\pm 10 \%$, both dependent on the mass ratio. The computed light curve based on these elements is shown in Fig. 3. This model takes into account a constant interval of brightness in the secondary minimum announced by Herczeg (1993) and confirmed by our observations $\left(d_{R} \simeq 35 \mathrm{~min}\right.$, see Fig. 4). The geometrical representation of V401 Cyg at phase 0.25 is displayed in Fig. 5 .

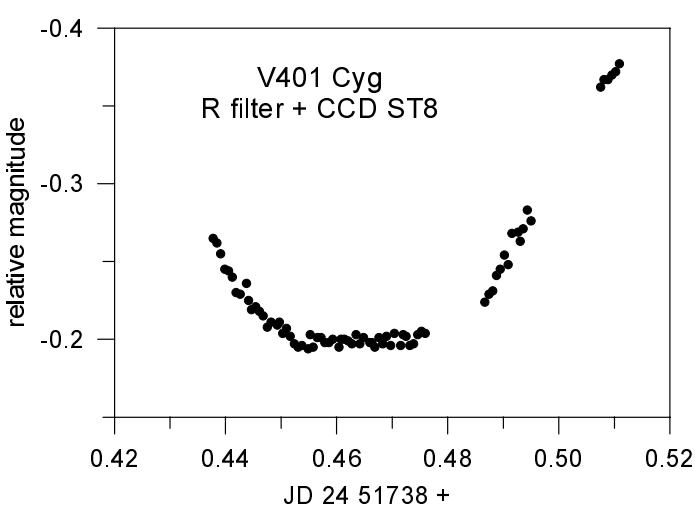

Fig. 4. A plot of the differential $R$-magnitudes of V401 Cyg obtained during the secondary eclipse at JD 2451738

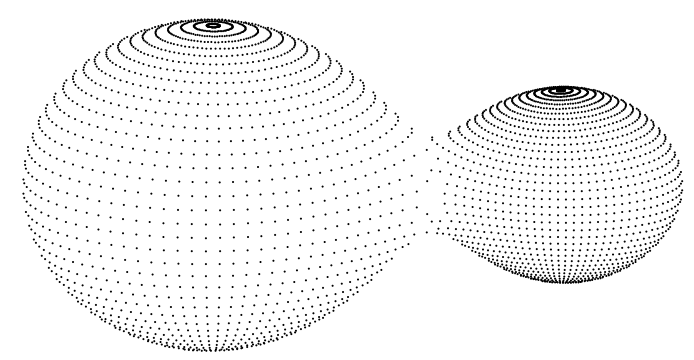

Fig. 5. Three-dimensional model of the contact system V401 Cyg at phase 0.25

\section{AD Phe}

The eclipsing binary AD Phe (also CD $-40^{\circ} 288$, CPD - 40 117, HIP 5955, PPM 276874, CSV 5906, $\mathrm{S} 7148, \mathrm{BV} 1260$, FL $90 ; \alpha_{2000}=1^{\mathrm{h}} 16^{\mathrm{m}} 38.1^{\mathrm{s}}, \delta_{2000}=$ $-39^{\circ} 42^{\prime} 31.3^{\prime \prime}, V_{\max }=10.29 \mathrm{mag}$ ) is a relatively seldom investigated southern system. It was discovered to be variable star photographically by Hoffmeister (1963). Strohmeier \& Bauernfeind (1969) published times of minimum light and determined the first light elements:

Pri. Min. = HJD $2428672.685+0.5132295 \cdot E$.

Several $U B V$ photoelectric times of minimum were obtained by Cerruti (1986) at Cerro Tololo Inter-American Observatry in Chile in August 1981. (In the title of this paper, AD Phe was erroneously designated as AG Phe). He improved light elements and found a reliable shortening of the period:

$$
\begin{aligned}
\text { Pri. Min. }= & \text { HJD } 2436781.4224 \\
& +0.37992632 \cdot E-0.64310^{-10} E^{2} .
\end{aligned}
$$

The precise $B V R I_{\mathrm{C}}$ photometry of $\mathrm{AD}$ Phe was obtained by McFarlane \& Hilditch (1987) at SAAO in October 1982. They derived five times of minimum light and calculated the revised ephemeris:

Pri. Min. = HJD $2446003.3683+0$ d $3799344 \cdot E$.

They also obtained a photometric solution, which was insensitive to the mass ratio. To our knowledge this star has not been studied spectroscopically since its discovery. 


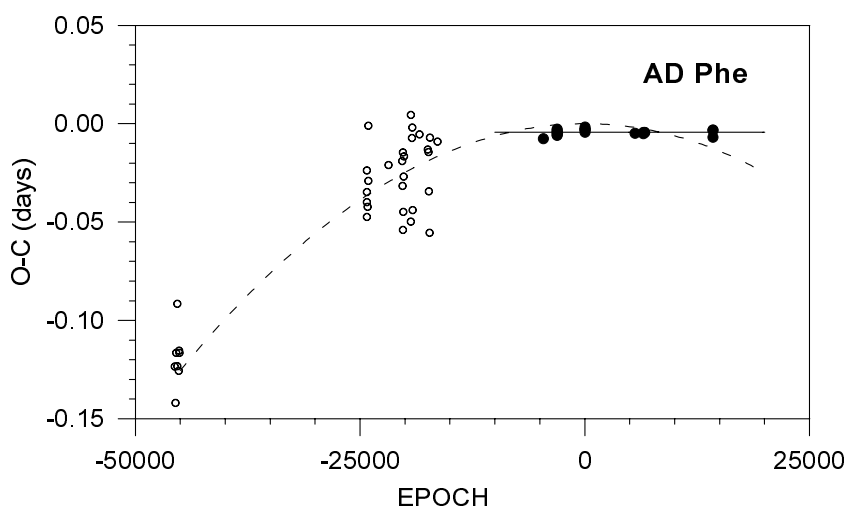

Fig. 6. $\mathrm{O}-\mathrm{C}$ diagram for $\mathrm{AD}$ Phe. The full line corresponds to our linear ephemeris, the dashed curve represents less probable parabolic light elements

More than 15 years have elapsed since its last study, thus AD Phe was also included in our photometric program at SAAO. Apart from the eclipses included in Table 1 by Cerruti (1986) we have adopted, for a new O-C diagram analysis, the minima given in McFarlane \& Hilditch (1987) and new timings resulting from the Hipparcos photometry. Using this data base, which includes 56 times of minima we improved the linear light elements after JD 2444800 Pri. Min. = HJD 24 45998.4292+0.37992454 $\cdot E$,

$$
\pm 0.0002 \pm 0.00000002
$$

which can serve also for the prediction of future times of minima. The corresponding $\mathrm{O}-\mathrm{C}$ diagram is plotted in Fig. 6.

\section{Y Sex}

The eclipsing binary Y Sex (also HD 87079, $\mathrm{BD}+01^{\circ}$ 2394, HIP 49217, PPM 156443, AN 358.1934, $\mathrm{FL} 1156, \quad \mathrm{P} 3375 ; \alpha_{2000}=10^{\mathrm{h}} 02^{\mathrm{m}} 48.0^{\mathrm{s}}, \quad \delta_{2000}=$ $\left.+01^{\circ} 05^{\prime} 40.3^{\prime \prime}, V_{\max }=9.97 \mathrm{mag} ; \mathrm{Sp} . \mathrm{F} 8\right)$ is a relatively bright and well-known A-type contact binary with an extremely low mass ratio. It was discovered to be a variable star by Hoffmeister (1934). Later, the system was observed photographically by Prikhodko (1947) and Gaposchkin (1953). The first photoelectric observations of Y Sex were presented by Tanabe \& Nakamura (1957) and Hill (1979) who derived a relatively low value of the mass ratio of the components $q=0.175$. Radial velocities were obtained by McLean \& Hilditch (1983), resulting in $q=0.18 \pm 0.03$, with good agreement with the photometrically determined value. Herczeg (1993), in his period study, derived these linear light elements:

Pri. Min. = HJD $2434445.9912+0 \mathrm{~d} 41981391 \cdot E$.

Recently, another period study of Y Sex was published

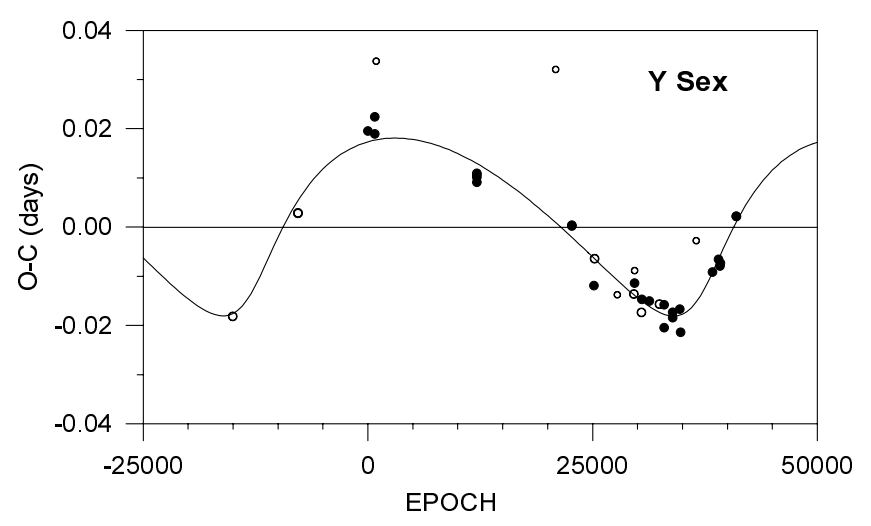

Fig. 7. O-C diagram for Y Sex. The curve corresponds to a third body orbit

by Qian \& Liu (2000). They presented the quadratic light elements

$$
\begin{aligned}
\text { Pri. Min. }= & \text { HJD } 2434445.9786 \\
& +0.41981527 \cdot E-3^{\mathrm{d}} .1410^{-11} E^{2}
\end{aligned}
$$

and interpreted the secular decrease of the period as mass transfer from the more to the less massive component or mass and angular momentum loss from the system. Surprisingly, the spectral type F8 given in Hill (1979) and mentioned also in Herczeg (1993) doesn't agree with the mass determination by Kaluzny $(1985), M_{\text {tot }}=0.81 M_{\odot}$. Apart from the eclipses included in Table 1 by Herczeg (1993) we have adopted, for a new determination of the period change of $\mathrm{Y}$ Sex, the numerous minima given by Agerer (1986, 1988, 1989, 1991, 1992, 1993), Agerer \& Hübscher (1998a, 1998b), Agerer et al. (1999) and Diethelm (1995). Using this data base, which includes 42 more reliable timings, we propose another explanation of the current $\mathrm{O}-\mathrm{C}$ diagram in Fig. 7. The sinusoidal variation of the period is remarkable and could be caused by a light-time effect. A preliminary analysis of the third body orbit gives the following parameters:

$P_{3}($ period $)=21050 \pm 50$ days, i.e. 57.6 years;

$T_{0}$ (time of periastron) $=$ JD $2450150 \pm 50$;

$A$ (semiamplitude $)=0.0181 \pm 0.0002$ day;

$e_{3}$ (eccentricity) $=0.52 \pm 0.06$

$\omega_{3}$ (length of periastron) $=320.0^{\circ} \pm 1.2^{\circ}$.

These values were obtained together with the new mean linear ephemeris

Pri. Min. = HJD $2434445.9695+0 \mathrm{~d} 41981485 \cdot E$,

$$
\pm 0.0002 \pm 0.00000001
$$

by the least squares method. Assuming a coplanar orbit $\left(i_{3}=90^{\circ}\right)$ and a total mass of the eclipsing pair according to the spectral type F8, $M_{1}+M_{2}=1.2 M_{\odot}$ (Harmanec 1988), we can obtain a lower limit for the mass of the third component $M_{3, \min }$. The value of the mass function is $f(M)=0.0120 M_{\odot}$, from which the minimum mass of the third body follows as $0.3 M_{\odot}$. A possible third component of spectral type M4-M5 with the bolometric 
Table 3. Eclipsing binaries with long-term continuos increase of the period

\begin{tabular}{cccccc}
\hline System & $\begin{array}{c}\text { Spectral } \\
\text { type }\end{array}$ & $\begin{array}{l}\text { Period } \\
\text { days }]\end{array}$ & $\begin{array}{c}\mathrm{d} P / \mathrm{d} E \\
{\left[10^{-10} \text { days/cycle }\right]}\end{array}$ & $\begin{array}{c}\mathrm{d} M / \mathrm{d} t \\
{\left[10^{-7} M \odot / \text { year }\right]}\end{array}$ & Reference \\
\hline AP Aur* & A2 & 0.56937 & 18.13 & 15.5 & Agerer \& Splittgerber (1993) \\
BX Dra* & A3 & 0.57903 & 11.12 & $* *$ & Agerer \& Dahm (1995) \\
XY Boo & F5V & 0.37055 & 6.20 & 1.34 & Molík \& Wolf (1998) \\
UZ Leo & A7 & 0.61804 & 6.07 & 1.30 & Hegedüs \& Jäger (1992) \\
V839 Oph & F8V & 0.40900 & 3.46 & 3.31 & Wolf et al. (1996) \\
AH Vir & K0V & 0.40752 & 2.66 & 0.76 & Demircan et al. (1991) \\
GO Cyg $*$ & B9+A0 & 0.71776 & 2.26 & 4.01 & Sezer et al. (1985), Rovithis (1997) \\
V401 Cyg & F0 & 0.58272 & 1.48 & 0.20 & this paper \\
44 i Boo & G2V+G2V & 0.26782 & 1.24 & 1.15 & Gherega et al. (1994) \\
DK Cyg & A8V & 0.47069 & 1.15 & 0.29 & this paper \\
CT Eri & F0 & 0.63420 & 1.02 & 0.38 & Lipari \& Sistero (1987) \\
\hline
\end{tabular}

Notes: ${ }^{*}$ EB type eclipsing binary, ${ }^{* *}$ data not available.

magnitude about +9.6 mag could be practically invisible in this system with an F8 primary $\left(M_{\text {bol }} \simeq+3.9 \mathrm{mag}\right)$. Therefore, new high-accuracy timings of this eclipsing binary are necessary in order to confirm the light-time effect in this system.

\section{Conclusions}

We derived updated light elements for four W UMa type eclipsing binaries by means of an $\mathrm{O}-\mathrm{C}$ diagram analysis. It was shown that the periods of all four systems exhibit different kind of changes.

In the case of DK Cygni, the derived value of the period increase is in excellent agreement with the result of Awadalla (1994). Our observations confirm the steady transfer of mass. V401 Cyg shows a relatively large increase in orbital period for W UMa-type binaries. Our mass transfer value is in good agreement with the previously obtained value by Herczeg (1993). Concerning the orbital and physical parameters, the system of V401 Cyg seems us to be similar to the system DK Cyg. According to the widely accepted theory, W UMa systems undergo periodic thermal relaxation oscillations ( $T R O$ theory, Lucy 1976; Flannery 1976). Each of these oscillations comprise a contact phase, when the mass flows into the primary component, and semidetached configuration, when the mass transfer is opposite. This cyclic exchange of mass has a period of $\simeq 10^{7}$ years. The list of other short-period eclipsing binaries with a long-term continuous increase in orbital period, ordered according to the value of $\mathrm{d} P / \mathrm{d} E$, is given in Table 3.

The period of $\mathrm{AD}$ Phe seems to be constant in the observed time interval. Our new observations at SAAO do not support the continuous shortening of the period proposed by Cerruti (1986). Further monitoring of this seldom-observed southern system could reveal other possible period variations.

Y Sex, as noticed by Kaluzny (1985), has one of the shortest orbital periods for such a spectral type (F8).
In this position in the period - colour diagram, one would expect an unevolved system with components with similar masses, but Y Sex seems to be evolved above ZAMS and its mass ratio is 5.5:1. The explanation of this paradox remains unknown. Period changes of Y Sex could be explained by a light-time effect caused by a third body on the eccentric orbit with a period of about 58 years. More high-accuracy timing of these systems are necessary in the future to enlarge the time span for a better analysis of period changes, especially for $\mathrm{Y}$ Sex, where the action of a third body in an eccentric orbit is predicted.

Acknowledgements. This study was supported by allocation of SAAO observing time, in part by the Grant Agency of the Czech Republic, grant No. 205/99/0255, and by the research plan J13/98: 113200004 Investigation of the Earth and the Universe. MW wishes to thank the staff at SAAO, Sutherland, for unfailing hospitality and help with the equipment and Dr. David Kilkenny for help with the photometric data reductions. We are also thankful to Mr. Franz Agerer, BAV, for timings of V401 Cyg from the Lichtenknecker's database. This research made use of the SIMBAD database, operated at CDS, Strasbourg, France.

\section{References}

Agerer F., 1986, BAV Mitteilungen No. 46, 2 Agerer F., 1988, BAV Mitteilungen No. 50, 4 Agerer F., 1989, BAV Mitteilungen No. 52, 6 Agerer F., 1990, BAV Mitteilungen No. 56, 4 Agerer F., 1991, BAV Mitteilungen No. 59, 7 Agerer F., 1992, BAV Mitteilungen No. 60, 8 Agerer F., 1993, BAV Mitteilungen No. 62, 6 Agerer F., Dahm M., 1995, IBVS No. 4266 Agerer F., Dahm M., Hübscher J., 1999, IBVS No. 4712 Agerer F., Hübscher J., 1995, IBVS No. 4222 Agerer F., Hübscher J., 1996, IBVS No. 4383 Agerer F., Hübscher J., 1998a, IBVS No. 4562 Agerer F., Hübscher J., 1998b, IBVS No. 4606 Agerer F., Splittgerber E., 1993, IBVS No. 3942 Awadalla N.S., 1994, A\&A 289, 137 
Binnendijk L., 1964, AJ 69, 157

Bradstreet D.H., 1993, Binary Maker 2.0, User Manual, Contact Software, Norristown, PA 19401-5505, U.S.A.

Cerruti M.A., 1986, Acta Astron. 35, 241

Demircan O., Derman E., Akalin A., 1991, AJ 101, 201

Diethelm R., 1995, BBSAG Bull. No. 108, 6

Flannery B.P., 1976, ApJ 205, 217

Gaposchkin S., 1953, Harvard Annals 113, No. 1

Gherega O., Farkas L., Horvath A., 1994, IBVS No. 4045

Guthnick P., Prager R., 1927, Astron. Nachr. 229, 445

Harmanec P., 1988, Bull. Astr. Inst. Czech. 39, 329

Hegedüs T., Bíró I.B., Borkovits T., Paragi Z., 1996, IBVS No. 4340

Hegedüs T., Jäger Z., 1992, PASP 104, 733

Herczeg T.J., 1993, PASP 105, 911

Hill G., 1979, Publ. Dom. Astrophys. Obs. Victoria 15, 297

Hinderer F., 1960, J. Obs. 43, 161

Hoffmeister C., 1929, Astron. Nachr. 236, 235

Hoffmeister C., 1934, Astron. Nachr. 253, 199

Hoffmeister C., 1963, Veroff. Sternw. Sonneberg 6, 1

Kaluzny J., 1985, Acta Astron. 35, 313

Kiss L.L., Kaszás G., Fürész G., Vinkó J., 1999, IBVS No. 4681

Lipari S.L., Sistero R.F., 1987, AJ 94, 792
Lucy L.B., 1976, ApJ 205, 208

Lurye M., 1947, Perem. Zvezdy 6, 208

McFarlane T.M., Hilditch R.W., 1987, MNRAS 227, 381

McLean B.J., Hilditch R.W., 1983, MNRAS 203, 1

Menzies J.W., Cousins A.W., Banfield R.M., Laing J.D., 1989, SAAO Circ. 13, 1

Molík P., Wolf M., 1998, IBVS No. 4630

Paparo M., Hamdy M.A., Jankovics I., 1985, IBVS No. 2838

Perryman M.A.C., 1997, The Hipparcos and Tycho Catalogues, ESA SP Ser. - 1200

Prikhodko A., 1947, Perem. Zvezdy 6, 136

Purgathofer A., 1964, Z. Astrophys. 59, 29

Qian S., Liu Q., 2000, A\&A 355, 171

Rovithis-Livaniou H., Rovithis P., Oprescu G., Dumitrescu A., Suran M.D., 1997, A\&A 327, 1017

Rucinski S.M., Lu W., 1999, AJ 118, 2451

Sezer C., Gülmen O., Güdür N., 1985, IBVS No. 2743

Spinrad H., 1959, PASP 71, 53

Strohmeier W., Bauernfeind H., 1969, IBVS No. 360

Tanabe H., Nakamura T., 1957, Ann. Tokyo Astron. Obs. 2nd Ser., 5

Wolf M., Šarounová L., Molík P., 1996, IBVS No. 4304 